OPEN ACCESS

Check for updates

\title{
Female authorship of covid-19 research in manuscripts submitted to 11 biomedical journals: cross sectional study
}

\author{
Angèle Gayet-Ageron, ${ }^{1,2,{ }^{*}}$ Khaoula Ben Messaoud, ${ }^{2, *}$ Mark Richards, ${ }^{3}$ Sara Schroter ${ }^{4}$
}

${ }^{1}$ Division of Clinical

Epidemiology, University

Hospitals of Geneva, Geneva, Switzerland

${ }^{2}$ Department of Health and Community Medicine, University of Geneva, Geneva, Switzerland

${ }^{3}$ Article Transfer Service, BMI

Publishing Group, London, UK

${ }^{4} \mathrm{BM}$, London, UK

*Contributed equally

Correspondence to:

A Gayet-Ageron

angele.gayet-ageron@hcuge.ch (or @AngeleGayet on Twitter:

ORCID 0000-0002-6164-9693)

Additional material is published online only. To view please visit the journal online.

Cite this as: $B M J$ 2021;375:n2288 http://dx.doi.org/10.1136/bmj.n2288

Accepted: 10 September 2021

\author{
ABSTRACT \\ OBJECTIVE \\ To describe prominent authorship positions held by \\ women and the overall percentage of women co- \\ authoring manuscripts submitted during the covid-19 \\ pandemic compared with the previous two years. \\ DESIGN \\ Cross sectional study.
}

SETTING

Nine specialist and two large general medical journals. POPULATION

Authors of research manuscripts submitted between 1 January 2018 and 31 May 2021.

\section{MAIN OUTCOME MEASURES}

Primary outcome: first author's gender. Secondary outcomes: last and corresponding authors' gender; number (percentage) of women on authorship byline in "pre-pandemic" period (1 January 2018 to 31 December 2019) and in "covid-19" and "noncovid-19" manuscripts during pandemic.

RESULTS

A total of 63259 manuscripts were included. The number of female first, last, and corresponding authors respectively were 1313 (37.1\%), 996 (27.9\%), and 1119 (31.1\%) for covid-19 manuscripts (lowest values in Jan-May 2020: 230 (29.4\%), 165 (21.1\%), and $185(22.9 \%)$ ), compared with $8583(44.9 \%)$, $6118(31.2 \%)$, and 7273 (37.3\%) for pandemic non-covid-19 manuscripts and 12724 (46.0\%), 8923 (31.4\%), and 10981 (38.9\%) for pre-pandemic manuscripts. The adjusted odds ratio of having a female first author in covid-19 manuscripts was $<1.00$ in all groups $(\mathrm{P}<0.001)$ compared with pre-pandemic (lowest in Jan-May 2020: 0.55, 98.75\% confidence interval 0.43 to 0.70 ). The adjusted odds ratio of

\section{WHAT IS ALREADY KNOWN ON THIS TOPIC}

Gender disparities have been widely reported in publication records, attainment of prominent authorship positions, contributions made to research, and access to academic promotion

During the covid-19 pandemic, researchers have put unprecedented effort into providing rapid knowledge on SARS-CoV-2 despite new challenges to working conditions

Analysis of submissions and publications in the opening months of the pandemic have indicated larger gender disparities than previously

\section{WHAT THIS STUDY ADDS}

Gender disparities in attainment of co-authorship and prominent authorship positions on submitted research were accentuated for covid-19 related

manuscripts early in the pandemic

These gender disparities in submitted manuscripts narrowed in the most recent pandemic period (Feb-May 2021) compared with the early pandemic (Jan-May 2020) having a woman as last or corresponding author was significantly lower for covid-19 manuscripts in all time periods (except for the two most recent periods for last author) compared with pre-pandemic (lowest values in Jan-May 2020: 0.74 (0.57 to 0.97) for last and 0.61 ( 0.49 to 0.77 ) for corresponding author). The odds ratios for pandemic non-covid-19 manuscripts were not significantly different compared with prepandemic manuscripts. The median percentage of female authors on the byline was lower for covid-19 manuscripts (28.6\% in Jan-May 2020) compared with pre-pandemic (36.4\%) and non-covid-19 pandemic manuscripts (33.3\% in Jan-May 2020). Gender disparities in all prominent authorship positions and the proportion of women authors on the byline narrowed in the most recent period (Feb-May 2021) compared with the early pandemic period (Jan-May 2020) and were very similar to values observed for pre-pandemic manuscripts.

\section{CONCLUSIONS}

Women have been underrepresented as co-authors and in prominent authorship positions in covid-19 research, and this gender disparity needs to be corrected by those involved in academic promotion and awarding of research grants. Women attained some prominent authorship positions equally or more frequently than before the pandemic on non-covid-19 related manuscripts submitted at some time points during the pandemic.

\section{Introduction}

Over recent decades, gender disparities in research production and contributions to published research have been demonstrated repeatedly. ${ }^{12}$ Women not only publish fewer research manuscripts overall than do their male counterparts but also, in biomedical research, less often occupy the highly sought after first and last authorship positions, with the potential for biased representation of female opinion. ${ }^{1-3}$ Women's reported contributions to published medical research also more frequently involve data collection, administration, and logistics, whereas men's contributions more often involve study conception and design, critical revision of the manuscript, or funding of the research; these differences in reported contributions were found to have persisted over a 15 year period. ${ }^{4}$

During the covid-19 pandemic, academic institutions, work places, schools, and childcare facilities have been forced to close globally, and women have been reported to be the most affected by the huge economic consequences. ${ }^{56}$ Researchers have been forced to work at home, many alongside family members, and take on additional responsibilities including daytime childcare and home schooling. All academic teaching had to be adapted for and delivered 
online. Despite these challenging lifestyle changes, we have seen an unprecedented increase in the number of research manuscripts submitted for publication. ${ }^{78}$ Some people argue that the consequences of the lockdown measures have been a heavier burden on women researchers with children than on men with children because home working, home schooling, and household tasks were mostly carried out by women. ${ }^{79}$ Some early studies looking at the impact of the opening months of the pandemic on published articles have shown underrepresentation of women overall and in prominent (first, last) authorship positions, specifically in articles related to covid-19. However, these studies either included a broad range of article types such as news and opinions as well as research publications, and/or selected data only on the early months of the pandemic in 2020, or were focused on an individual journal, medical specialty, or geographic region. ${ }^{810-18} \mathrm{~A}$ more recent large study of first authorship attainment, in PubMed indexed life sciences articles with the term "covid" in the title and/or abstract published up to January 2021, found an overall gender gap in the early periods of the pandemic (1 February to 31 May 2020), but with a trend backwards to expected values over time in various medical disciplines. ${ }^{14}$ Two further studies showed no gender disparities in publications in the early months of the pandemic, but these were undertaken only in American journals or in a single specialty, medical imaging. ${ }^{15} 19$

Three studies showed gender inequalities in submitted research, but all of these focused on the early months of the pandemic (up to May 2020), ${ }^{101819}$ and two of them assessed submissions to a single specialist journal. ${ }^{1019}$ An analysis of the corresponding authors of submissions to The Journal of Pediatrics found that men submitted a significantly larger proportion of original articles compared with women in April-May 2020 than in April-May 2019. ${ }^{10}$ An analysis of submissions to the American Journal of Public Health showed that the increase in submissions from male corresponding authors was almost four times higher than for women, and the proportion of female corresponding authors of covid-19 manuscripts was lower than the proportion for submissions in the pre-pandemic period. ${ }^{20}$ The third study explored manuscript submissions in Elsevier journals during the pandemic (February-May 2020) compared with February-May 2018-19 and found that, overall, women submitted proportionately fewer research manuscripts than did men, and this was more prominent for the health sciences and for manuscripts related to covid-19, but the study did not look at the attainment of prominent authorship positions. ${ }^{21}$

The objective of this cross sectional study was to describe the attainment of prominent authorship positions (first, last, and corresponding author) and the overall percentage of female authors on research manuscripts submitted to 11 biomedical journals during the covid-19 pandemic (1 January 2020 to 31 May 2021) compared with the previous two years (2018-19). We hypothesised that gender disparities in research production during the covid-19 pandemic would be more pronounced at the beginning of the pandemic when public health measures were applied almost everywhere compared with a two year prepandemic period. We also hypothesised that gender disparities would be particularly pronounced in research production conceived and conducted during the pandemic-that is, among manuscripts in which covid-19 was mentioned compared with manuscripts not related to covid-19.

\section{Methods}

\section{Study design and settings}

This cross sectional study is ancillary to a broader research project, the ATHENA study, assessing whether gender bias exists in the editorial decision making process. Both studies are being conducted under a confidentiality agreement between BMJ Publishing Group and the medical school of Geneva University represented by the Department of Health and Community Medicine.

Editors of all BMJ Publishing Group journals were invited to take part in the ATHENA study. We selected a subsample of 11 journals of varying size and impact factor from a range of biomedical disciplines, including two large general medical journals (The BMJ and BMJ Open) and nine specialist journals (BMJ Global Health; Occupational and Environmental Medicine; Heart; Gut; Thorax; Journal of Neurology, Neurosurgery and Psychiatry; Postgraduate Medical Journal; Sexually Transmitted Infections; and Tobacco Control). As the focus was on research submissions, participating journals needed to have clear submission categories for original research, protocols, and/or systematic reviews on ScholarOne (the manuscript submission system) to be eligible to take part, as well as being published by BMJ Publishing Group for at least eight years to enable comparisons over time.

\section{Participants and data source}

Using the ScholarOne article types selected by authors on submission, we selected all research manuscripts (original research, systematic reviews and/or metaanalysis), including research study protocols, that were submitted for publication to one of the 11 participating journals between 1 January 2018 and 31 May 2021. We excluded all other article types on the basis of the ScholarOne submission categories indicated by submitting authors. We identified manuscripts that were submitted to one of the 11 journals and then transferred to another of these journals in the study period by using "manuscript transfer ID," "transferring journal name," "receiving journal name," and "manuscript title" and included only the first submission. We extracted data from the ScholarOne manuscript submission systems.

\section{Factors and outcomes}

We defined two submission periods: the "pre-pandemic period” (1 January 2018 to 31 December 2019) and the "pandemic period" (1 January 2020 to 31 May 2021). We further defined four time periods during 
the pandemic period corresponding roughly to the evolution of the pandemic worldwide: Jan-May 2020, Jun-Sep 2020, Oct 2020-Jan 2021, and Feb-May 2021. In total, we had five time-periods and nine different groups (including covid-19 versus non-COVID-19 manuscripts in the pandemic period).

To identify manuscripts describing studies on patients with covid-19 or studies on the impact of covid-19 on health services and policies, we further categorised manuscripts submitted in the pandemic period as "covid-19" manuscripts or "pandemic noncovid-19" manuscripts on the basis of presence/ absence of the following keywords in the title and/ or abstract variables: "covid," "covid-19," "SARSCoV-2,” "coronavirus," "2019-nCov,” "coronavirinae,” "coronaviridae," "hCoV-19," or "SARS and virus." To estimate the accuracy of our search strategy for covid-19 relevant manuscripts, two independent raters (AGA and SS) assessed the titles and abstracts of a random sample of 300 manuscripts identified as related to covid-19 and indicated their agreement.

For each manuscript, we collected two levels of information. At the manuscript level, we recorded the following variables: journal name, manuscript ID, title, abstract, date of original submission, number of co-authors on the byline, date of transfer to another journal where applicable, name of the receiving journal, and editorial decision by 11 August 2021. At the author level, we recorded the following variables: author ID; author's first, middle, and last names; author's salutation; author's country; rank authorship order on the article byline (several co-authors could hold the first authorship position, but we used only first declared author), and corresponding authorship status.

Our primary outcome was the first author's gender. Secondary outcomes were the last and corresponding author's gender and the percentage of women among all co-authors on the article byline. We evaluated primary and secondary outcomes among nine groups: pre-pandemic and either covid-19 or pandemic noncovid-19 manuscripts in Jan-May 2020, Jun-Sep 2020, Oct 2020-Jan 2021, and Feb-May 2021.

\section{Gender determination}

We determined gender by using a sequential four step procedure. Firstly, we used both first name and country to search in Gender API (https://gender-api.com/en) website. Gender API provides gender determination with an accuracy probability from $50 \%$ to $100 \%$ (under $50 \%$ an unknown status is attributed), gives the number of samples retrieved to search for the information, ensures that the level of misclassification is around $5 \%$, and has the highest coverage on the variability in name origins. ${ }^{22}$ We set gender accuracy above $80 \%$ as the minimum criterion for inclusion in the primary gender determination. Secondly, for all authors with undetermined or unknown gender based on the above, we used the authors' given middle names and country to search in Gender API. Thirdly, we used the online service genderize.io (http://genderize.io) to determine gender on the basis of first names and middle names, also using an accuracy above $80 \%$ as the minimum criterion. Fourthly, for still undetermined gender, we used the authors' salutation and attributed with 100\% accuracy male gender to "Mr" or "M" and female gender to "Miss," "Mrs," and "Ms." Where none of these methods determined the gender, we recorded the gender as undetermined.

\section{Statistical analysis}

Our sample size was fixed (n=63 259 manuscripts, including 34222 pre-pandemic manuscripts, 4447 covid-19 manuscripts, and 24590 pandemic noncovid-19 manuscripts); these numbers allowed us to detect at least a 3.93\% decrease in the proportion of female first authors between pre-pandemic and covid-19 related submissions with 90\% study power and 5\% type I error (two sided), taking into account that the data were clustered within the country of affiliation of first authors nested within journals (we took mean cluster size at 30 and intraclass correlation at 0.05 , leading to a design effect of 2.45).

We described continuous variables by mean (standard deviation) or median (interquartile range) and presented categorical variables as frequencies and relative proportions. We plotted the daily number of manuscripts submitted across time by using time series smoothed curves with a moving average by seven days.

We described outcomes and manuscripts' characteristics in the nine groups. We compared proportions of manuscripts submitted to generalist versus specialised journals among time groups by using a $\chi^{2}$ test. We used a Kruskal-Wallis non-parametric test for comparison of total number of co-authors among time groups. For outcomes, we reported $\mathrm{P}$ values assessing outcomes across the nine groups from the univariate models described below.

We used bivariate mixed effects logistic regression models to test associations between groups (covid-19 and pandemic non-covid-19 versus pre-pandemic manuscripts) and first author's gender by journal specialty. We assessed the probability of the first author being a woman across the nine groups, independently of last author's gender, total number of co-authors, and categories of journal (generalist versus specialist). We further adjusted for the level of missing gender determination at country level $(<25 \%, 25-50 \%$, and $\geq 50 \%$ versus none missing). We also compared the chance of being a female first author on covid-19 and pandemic non-covid-19 manuscripts versus prepandemic manuscripts. We graphically verified the linearity assumption of logit for continuous variables. We used mixed effects logistic regression models with first author's gender as the dependent variable (model 1). To account for some clustering in the data the journal and country of affiliation levels, we included nested random factors on the intercept by using the country of affiliation within journals. To increase the precision of estimates, we restricted the analyses to countries with at least three submissions per reported group. We used similar models to assess the probability of the last author (model 2) and corresponding author 
(model 3) being a woman. For model 3, we also adjusted for the position of the corresponding author on the article byline (last, first, or other position). We interpreted global $P$ values as significant at the adjusted cut-off 0.0125 , using the Bonferroni method to take into account multiple comparisons (0.05/4 outcomes). We ran these regression models on complete cases and reported adjusted odds ratios, 98.75\% confidence intervals, and $\mathrm{P}$ values. We did sensitivity analyses with accuracy of gender determination above $95 \%$. We also did post hoc sensitivity analyses to quantify the potential of missing gender determination (for model 1 , model 2, and model 3) by using multiple imputation for missing at random, ${ }^{23}$ with $\mathrm{M}=20$ imputations and adjustment of the imputation model for author's country, nine time groups, and journal specialty.

For the assessment of the percentage of women among all co-authors across groups (model 4), we reported results only for manuscripts that had gender determined for at least $80 \%$ of all co-authors. Firstly, we plotted the number of women co-authors among the last author's region of affiliation (as a proxy of research origin), to visualise distribution (supplementary figure C). We observed two different patterns between authors with affiliation in Europe, North America, Latin America, or Oceania (region 1) and those with affiliation in Africa, China, or the rest of Asia (region 2). For manuscripts from region 1, we further used a mixed effects Poisson regression model with total number of co-authors on the byline and with determined gender as offset, nested random factors on the intercept using the last author's country of affiliation within journals, and we restricted the analyses to countries with at least three submissions per reported group (model 4A). For manuscripts from region 2, we used a second mixed effects Poisson regression model with nested random factors on the intercept and using last author's geographical region (and not country) of affiliation within journals (model 4B). We adjusted both models for the type of journal (generalist versus specialist) and for the level of missing gender determination at country level. We verified the goodness of fit of the Poisson models by a visual inspection of Anscombe's residuals. We reported incidence rate ratios, 98.75\% confidence intervals, and $P$ values.

We interpreted global $\mathrm{P}$ values as significant at the adjusted cut-off of 0.0125 , with only $\mathrm{P}<0.0125$ yielding a confidence interval not crossing the null value. We used Stata 16 intercooled and $\mathrm{R}$ software (version 3.3.1) for analyses.

\section{Patient and public involvement}

We partnered and co-authored with the chair of the BMJ's LGBTQ+ network (MR). MR contributed to almost every aspect of the study (data collection, gender determination, manuscript revision, final approval).

\section{Results}

Studied population

A total of 63259 manuscripts were included in our study among the 75731 manuscripts submitted for publication to the 11 journals (supplementary figure A), with 551341 authors across all included manuscripts (supplementary table A). Corresponding authorship overlapped with first and last authorship in $30703(48.5 \%)$ and 25039 (39.6\%) manuscripts. Gender was determined for $445052(80.7 \%)$ of authors (supplementary table A). Supplementary figure B shows the gender determination process. We found a high level of agreement $(\kappa=0.95)$ between raters on the identification of covid-19 manuscripts.

\section{Descriptive analysis}

Manuscript submissions during 2020 increased by $18.6 \%$ compared with 2019 and by $23.3 \%$ compared with 2018 (fig 1); 15.3\% (4447/29037) of submissions during the pandemic were covid-19 related manuscripts that were submitted as a priority to generalist journals (table 1). We observed similar proportions for editorial decisions (we did not consider manuscripts with no decision yet here to calculate proportions) on manuscripts submitted before the pandemic $(n=34202)$ and on pandemic non-covid-19 manuscripts $(n=20668)$ : respectively, 24964 (73.0\%) versus 15468 (74.8\%) were rejected, $8884(26.0 \%)$ versus 4855 (23.5\%) were accepted, and $354(1.0 \%)$ versus $345(1.7 \%)$ were still under revision by 11 August 2021 (table 1; supplementary table A). Among covid-19 manuscripts ( $n=3740)$, we observed a higher proportion of rejected manuscripts (83.8\%; $n=3134)$ compared with pre-pandemic and pandemic non-covid-19 manuscripts, as well as a lower proportion of accepted manuscripts (15.2\%; $\mathrm{n}=568)$ and manuscripts under revision $(1.0 \% ; \mathrm{n}=38)$ (table 1; supplementary table A). Total numbers of coauthors on the manuscript byline were significantly higher by a mean of 2 additional co-authors in covid-19 manuscripts compared with pre-pandemic and pandemic non-covid-19 manuscripts.

Overall, we observed an underrepresentation of women in prominent authorship positions (first 22620 (44.9\%), last 16037 (31.1\%), and corresponding $19373(37.7 \%)$ ), and among all co-authors (median percentage of female co-authors 35.7\%). The number of female first, last, and corresponding authors respectively were $1313(37.1 \%), 996$ (27.9\%), and 1119 (31.1\%) for covid-19 manuscripts (lowest values in Jan-May 2020: 230 (29.4\%), 165 (21.1\%), and $185(22.9 \%)$ ), compared with 8583 (44.9\%), 6118 (31.2\%), and 7273 (37.3\%) for pandemic non-covid-19 manuscripts and 12724 (46.0\%), 8923 (31.4\%), and 10981 (38.9\%) for pre-pandemic manuscripts (supplementary table A). The proportions of female first, last, and corresponding authors were significantly lower in covid-19 manuscripts $(-8.9 \%,-3.5 \%$, and $-7.8 \%$, respectively) in comparison with pre-pandemic manuscripts (supplementary table A). The drop in proportions of female first, last, and corresponding authors in comparison with pre-pandemic was the greatest in Jan-May $2020(-16.6 \%,-10.3 \%$, and $-16.0 \%$, respectively; table 1 ), the deepest being in March-April 2020 (fig 2). These gender differences 


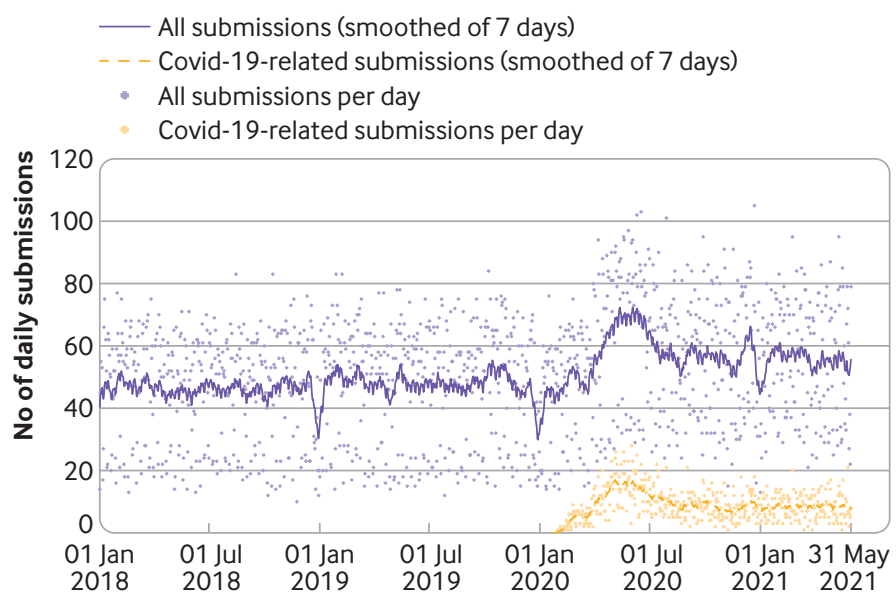

Fig 1 | Curves (using smoothing parameter of seven days) of total number of research manuscripts and covid-19 manuscripts submitted per day between 1 January 2018 and 31 May 2021 to 11 participating journals from BMJ Publishing Group

narrowed in the most recent period compared with the early pandemic period and were very similar to values observed for pre-pandemic manuscripts; for last authorship, the proportion of women was even higher in the two last periods of 2021 than pre-pandemic. The proportions of female first, last, and corresponding authors in pandemic non-covid-19 manuscripts were very similar to pre-pandemic manuscripts (table 1; supplementary table A; fig 2). The percentage of female authors on the byline was lower in covid-19 manuscripts in comparison with pre-pandemic manuscripts (supplementary table A); the drop was the greatest in Jan-May 2020 (median 28.6\% female co-authors compared with $36.4 \%$ in pre-pandemic; table 1). The odds of the first author being a woman were significantly lower in covid-19 manuscripts compared with pre-pandemic manuscripts for most specialties (supplementary table B). For generalist journals, representing the majority of the dataset, the odds of the first author being a woman was 0.74-fold (98.75\% confidence interval 0.68 to 0.81 ) lower in covid-19 manuscripts compared with pre-pandemic manuscripts; it was significantly higher by 1.05 -fold (1.00 to 1.11) in non-covid-19 manuscripts compared with pre-pandemic manuscripts.

In all groups, most authors had a European affiliation, with some variations among first, last, and corresponding authors (supplementary table $\mathrm{C}$ ), except for covid-19 manuscripts submitted in Jan-May 2020, in which the proportions of authors with a Chinese affiliation were the highest (474 (43.0\%), 451 (40.9\%), and $460(41.7 \%)$ of first, last, and corresponding authors compared with 6479 (18.9\%), 6192 (18.1\%), and 6334 (18.5\%) in pre-pandemic manuscripts). By June-Sept 2020, the distribution of authors' affiliations returned to a pattern similar to that seen in the prepandemic period, with European affiliations being the most frequent. The proportions of missing gender for first, last, and corresponding authors were the highest for authors with a Chinese affiliation (supplementary table D). Among authors with determined gender, we observed gender bias against women in most countries, except in Latin America and Oceania where first authors were women in respectively 646 (54.4\%) and 1644 (57.9\%) (supplementary table D). Men predominantly occupied the last author and the corresponding author position in all regions.

\section{Multivariable analysis}

Primary outcome: first authorship

The probability of having a female first author significantly decreased across groups during the pandemic $(\mathrm{P}<0.001$; model 1 , table 2$)$; it was the lowest in Jan-May 2020: odds ratio 0.55 (0.43 to 0.70) in covid-19 manuscripts. The odds of female first authors decreased (adjusted odds ratio 0.67, 0.60 to 0.75 ) among covid-19 manuscripts compared with prepandemic manuscripts (model 1, supplementary table E). Having a female last author was associated with a higher probability of having a woman as first author (adjusted odds ratio 1.84, 1.74 to 1.94; model 1, table 2). Total number of co-authors on the article byline and journal type were not significantly associated with first author's gender (table 2).

\section{Last authorship}

The probability of having a woman as last author significantly decreased across groups during the pandemic ( $\mathrm{p}=0.01$; model 2, table 2); it was lowest in Jan-May 2020: odds ratio 0.74 (0.57 to 0.97) in covid-19 manuscripts. The odds ratio was not significantly lower for covid-19 than pre-pandemic manuscripts: adjusted odds ratio 0.93 (0.82 to 1.04) (model 2, supplementary table E). Having a higher number of co-authors was associated with a lower probability of having a woman as last author (adjusted odds ratio $0.98,0.98$ to 0.99 ). Journal type was not significantly associated with the probability of having a female last author.

\section{Corresponding authorship}

The probability of having a female corresponding author significantly decreased across groups during the pandemic ( $\mathrm{p}<0.001$; model 3 , table 2 ); it was lowest in Jan-May 2020: odds ratio 0.61 (0.49 to 0.77 ) in covid-19 manuscripts. The odds ratio was significantly decreased for covid-19 manuscripts compared with pre-pandemic manuscripts (adjusted odds ratio 0.72, 0.65 to 0.80 ) (model 3, supplementary table E). Being the first or a middle rank author compared with last author was associated with a higher probability of having a woman as the corresponding author: respective adjusted odds ratios 1.71 (1.61 to 1.81) and 1.26 (1.16 to 1.38). Having a higher number of co-authors was associated with a lower probability of having a female corresponding author (adjusted odds ratio $0.99,0.98$ to 0.99 ). Journal type was not significantly associated with the probability of having a woman corresponding author.

We confirmed the same results for first, last, and corresponding authors in our sensitivity analyses using an accuracy above $95 \%$ for gender determination 


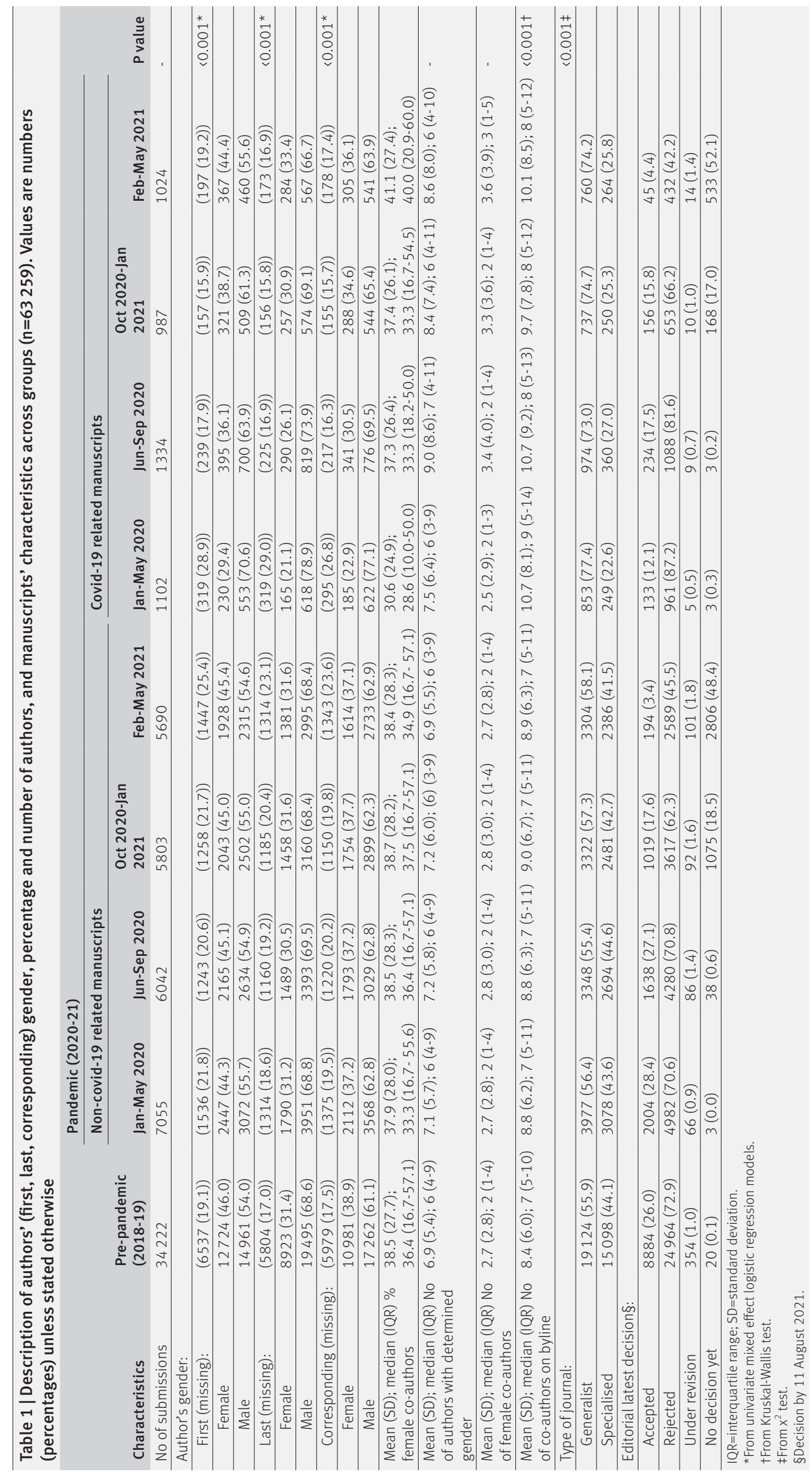



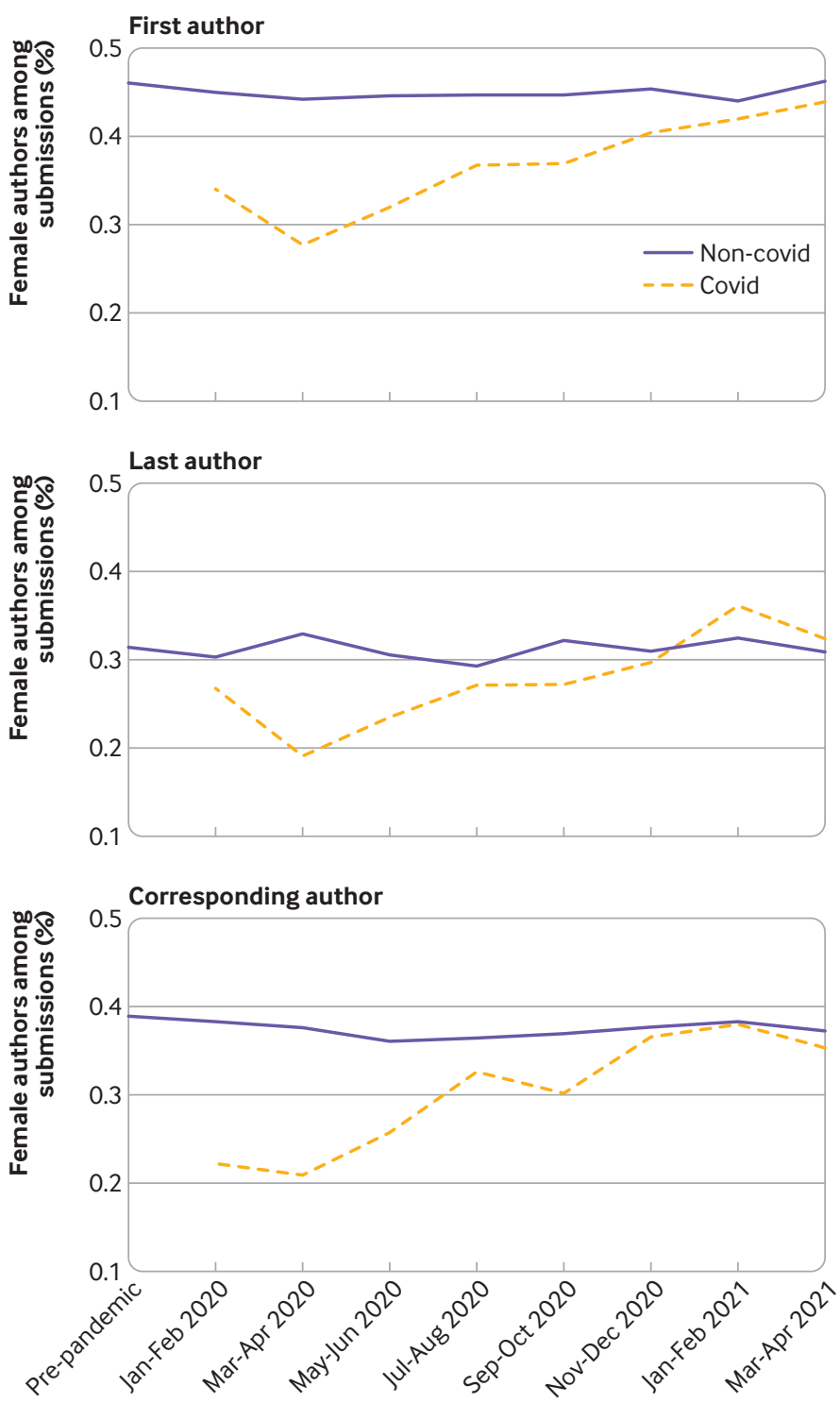

Fig 2 | Proportion of female first, last, and corresponding authors across two month periods between 1 January 2018 and 31 May 2021 among covid-19 and non-covid-19 manuscripts submitted to 11 participating journals from BMJ Publishing Group (supplementary table F) (except for the association of female last author, which was no longer associated with the groups). We observed similar results as previously when we used multiple imputation (supplementary table $\mathrm{H}$ ). Having a woman as last or first author was associated with a higher probability of having a woman as first or last author, respectively, and multiple imputation provided reinforced associations.

\section{Percentage of female co-authors}

We observed two different patterns for distributions of numbers (percentages) of female co-authors on manuscripts with an affiliation for the last author in Africa, China, or the rest of Asia and those in Europe, North America, Latin America, or Oceania (supplementary figure C). We further present two stratified multivariable models (models 4A and 4B, table 3) assessing the incidence rate ratio of female co-authors across groups. The incidence rate ratio was significantly decreased by 0.95 -fold (0.93 to 0.98) across groups among covid-19 manuscripts compared with pre-pandemic manuscripts (model 4A, supplementary table G) in Europe, North America, Latin America, or Oceania; it was lowest in Jan-May 2020: 0.88 (0.82 to 0.94) (model 4A, table 3). The number of female co-authors on the article byline was not significantly associated with the type of journal (specialised versus generalist).

For the analysis of manuscripts with the last author's affiliation in Africa, China, or the rest of Asia, we used a smaller sample owing to the exclusion of manuscripts with more than 20\% undetermined gender (model 4B, table 3). The number of female co-authors decreased (adjusted odds ratio 0.94, 0.90 to 0.98) across groups among non-covid-19 manuscripts compared with prepandemic manuscripts (model 4B, supplementary table $\mathrm{G}$ ), with lower incidence rate ratios at two time points (Jun-Sep 20 and Oct 20-Jan 21) compared with pre-pandemic (model 4B, table 3 ). The number of female authors was not significantly associated with the type of journal.

\begin{tabular}{|c|c|c|c|c|c|c|}
\hline \multirow[b]{2}{*}{ Independent variables } & \multicolumn{2}{|c|}{ First author $($ model 1$) *(n=43319)$} & \multicolumn{2}{|c|}{ Last author $($ model 2$) *(n=43297)$} & \multicolumn{2}{|c|}{ Corresponding author $(\text { model } 3)^{\star}(n=50896)$} \\
\hline & Odds ratio $(98.75 \% \mathrm{Cl})$ & Pvaluet & Odds ratio $(98.75 \% \mathrm{Cl})$ & $P$ valuet & Odds ratio $(98.75 \% \mathrm{Cl})$ & Pvaluet \\
\hline Groups (reference=pre-pandemic): & & $<0.001$ & & 0.01 & & $<0.001$ \\
\hline Jan-May 2020 covid-19 & $0.55(0.43$ to 0.70$)$ & $<0.001$ & $0.74(0.57$ to 0.97$)$ & 0.005 & 0.61 (0.49 to 0.77$)$ & $<0.001$ \\
\hline Jan-May 2020 non-covid-19 & $0.96(0.88$ to 1.05$)$ & 0.24 & $0.99(0.91$ to 1.09$)$ & 0.80 & $0.98(0.90$ to 1.06$)$ & 0.56 \\
\hline Jun-Sep 2020 covid-19 & 0.65 (0.54 to 0.78$)$ & $<0.001$ & 0.84 (0.68 to 1.03$)$ & 0.03 & $0.70(0.59$ to 0.84$)$ & $<0.001$ \\
\hline Jun-Sep 2020 non-covid-19 & $1.02(0.93$ to 1.12$)$ & 0.54 & $1.00(0.91$ to 1.11$)$ & 0.92 & $1.00(0.92$ to 1.09$)$ & 0.94 \\
\hline Oct 2020-Jan 2021 covid-19 & $0.68(0.55$ to 0.84$)$ & $<0.001$ & $1.01(0.81$ to 1.25$)$ & 0.93 & $0.77(0.63$ to 0.94$)$ & 0.001 \\
\hline Oct 2020-Jan 2021 non-covid-19 & $0.99(0.91$ to 1.09$)$ & 0.93 & $1.06(0.96$ to 1.18$)$ & 0.11 & $1.06(0.97$ to 1.15$)$ & 0.12 \\
\hline Feb-May 2021 covid-19 & $0.82(0.67$ to 1.01$)$ & 0.02 & $1.10(0.89$ to 1.37$)$ & 0.25 & $0.83(0.68$ to 1.01$)$ & 0.02 \\
\hline Feb-May2021 non-covid-19 & $1.01(0.92$ to 1.11$)$ & 0.79 & 1.07 (0.97 to 1.19$)$ & 0.10 & $1.02(0.93$ to 1.11$)$ & 0.66 \\
\hline Female first author (reference=male) & - & - & 1.84 (1.74 to 1.94$)$ & $<0.001$ & - & - \\
\hline Female last author (reference $=$ male) & $1.83(1.73$ to 1.94$)$ & $<0.001$ & - & - & - & - \\
\hline Author type (reference=last): & & & & & & $<0.001$ \\
\hline First & - & - & - & - & 1.71 (1.61 to 1.81$)$ & $<0.001$ \\
\hline Middle rank & - & - & - & - & $1.26(1.16$ to 1.38$)$ & $<0.001$ \\
\hline No of authors on article byline & $0.99(0.99$ to 1.00$)$ & 0.24 & $0.98(0.98$ to 0.99$)$ & $<0.001$ & 0.99 (0.98 to 0.99$)$ & $<0.001$ \\
\hline Specialist journal (reference=generalist) & $1.01(0.66$ to 1.54$)$ & 0.97 & $0.99(0.53$ to 1.84$)$ & 0.96 & $0.95(0.54$ to 1.68$)$ & 0.83 \\
\hline
\end{tabular}




\begin{tabular}{|c|c|c|c|c|}
\hline \multirow[b]{2}{*}{ Independent variables } & \multicolumn{2}{|c|}{$\begin{array}{l}\text { No of female co-authors (model } 4 A) * \\
(n=32196)\end{array}$} & \multicolumn{2}{|c|}{$\begin{array}{l}\text { No of female co-authors (model 4B) } t \\
(n=7972)\end{array}$} \\
\hline & IRR $(98.75 \% \mathrm{CI})$ & $P$ value $\neq$ & IRR $(98.75 \% \mathrm{CI})$ & P value $\neq$ \\
\hline Groups (reference=pre-pandemic): & & $<0.001$ & & 0.006 \\
\hline Jan-May 2020 non-covid-19 & $1.00(0.98$ to 1.03$)$ & 0.73 & $0.95(0.89$ to 1.02$)$ & 0.06 \\
\hline Jun-Sep 2020 covid-19" & $0.94(0.90$ to 0.98$)$ & 0.001 & $0.95(0.84$ to 1.08$)$ & 0.34 \\
\hline Jun-Sep 2020 non-covid-19 & $1.02(0.99$ to 1.04$)$ & 0.09 & 0.93 (0.87 to 0.99) & 0.01 \\
\hline Oct 2020-Jan20 21 covid-19 & $0.96(0.91$ to 1.01$)$ & 0.06 & 0.95 (0.82 to 1.09$)$ & 0.35 \\
\hline Oct 2020-Jan 2021 non-covid-19 & $1.02(0.99$ to 1.04$)$ & 0.12 & $0.93(0.86$ to 0.99$)$ & 0.009 \\
\hline
\end{tabular}

\section{Discussion}

Analysis of research manuscripts submitted to 11 journals from the BMJ Publishing Group showed a dramatic increase during the covid-19 pandemic in comparison with the previous two years. At the same time, gender inequalities in the authorship of these submissions increased. Throughout the pandemic (up to Jan-May 2021), women were less represented in co-authorship and prominent authorship positions on research related to covid-19 than in research submissions before the pandemic. The widest gender gap was between 1 January and 31 May 2020 compared with before the pandemic, and this was most pronounced for first and corresponding authors. For the two last periods (Oct 2020-Jan 2021 and Feb-May 2021), these gender disparities narrowed compared with the early pandemic period and were very similar to observed values for the pre-pandemic period. The median percentage of female authors on the byline was lower for covid-19 manuscripts (28.6\% in JanMay 2020) compared with pre-pandemic manuscripts (36.4\%). Having a female last author was associated with a higher probability of having a woman as first author; having more co-authors on the byline was associated with a lower chance of having a woman as last or corresponding author. Additionally, having a female last author was associated with a lower probability that this woman was also the corresponding author. Most submitted manuscripts were from European affiliated authors, except in Jan-May 2020 when Chinese affiliated authors were predominant.

\section{Comparison with other studies}

Our findings are broadly in line with the three previous studies of gender disparities in research submissions during the early months of the pandemic (up to May 2020). These studies also showed that women submitted proportionately fewer articles overall (from $-0.9 \%$ up to $-24 \%$ ) than men in the early months of the pandemic and authored fewer covid-19 related articles overall $(-7.6 \%$ fewer female than male co- authors in health and medicine). ${ }^{8102021}$ Bell et al's smaller study of submissions to American Public Health showed the proportions of first and corresponding authorships to be significantly higher for men $(36 \%$ and $42 \%$ respectively) than women (12\% and $11 \%$ respectively) between April and May 2020 compared with April to May 2019, but with variation by country. Similarly to us, Bell et al also reported that less than a third of covid-19 related submissions had female corresponding authors. ${ }^{20}$ Williams et al's analysis of the increased volume of submissions to the American Journal of Pediatrics during January to May 2020 found that the greatest increase was in international male corresponding authors. ${ }^{10}$ Like Squazzoni et al, who looked at the number of manuscripts submitted by female and male authors (regardless of authorship position) in the opening months of the pandemic, we found a lower proportion of female than male co-authors in the covid-19 manuscripts (median $33.3 \%$ ), which was even lower in January to May 2020 (median 28.6\%), compared with the pre-pandemic period (median $36.4 \%$ ). ${ }^{21}$ We found similar results to a smaller study of articles, news, and opinion pieces on covid-19 published before 1 May 2020, reporting that $31 \%$ (95\% confidence interval $29 \%$ to $33 \%$ ) of articles were co-authored by women during the first semester of $2020 .^{8}$ In this study, Pinho-Gomes reported even more pronounced gender disparities than in our study, with $29 \%$ of first and $26 \%$ of last authors being women. However, these values may be lower than ours owing to the inclusion of a large range of article types other than research, use of a different method to distinguish covid-19 related articles, and a focus on publications in the opening months of the pandemic when working patterns will have been most disrupted. Lerchenmüller et al's longitudinal study of covid-19 related articles published up to 31 January 2021 found that the proportion of women in first and last authorship positions started to trend backwards to values observed before the pandemic. ${ }^{14}$ We observed a similar trend regarding first, last, and, additionally, 
corresponding authorship positions over a longer study period (up to 31 May 2021).

Similarly to others, we found some association between authorship position on the byline and the geographical affiliation of authors. ${ }^{342024-27}$ Bell et al reported that the increase in submission rates between the pre-pandemic and pandemic periods was greater for male than for female first and corresponding authors affiliated to a non-US country versus the US. ${ }^{20}$ Pinho-Gomes et al reported that the percentage of female co-authors on published research was lowest in Africa and highest in Oceania. ${ }^{8}$ Lerchenmüller et al outlined deeper gender disparities in first authorship positions at the beginning of the pandemic with some variations according to geographical area, with a greater gender gap in Africa and lesser one in Asia compared with our findings. ${ }^{14}$ Beyond scientific production, in many countries very few women researchers work in biomedicine and even fewer in key positions, as women's place in society, and in science, depends on the socio-cultural context of the country. ${ }^{25}$ Therefore, meta-research is needed to explore these countries' academic systems and research practices with the goal of assessing policies that might explain these differences.

\section{Strengths and limitations of study}

Although our study included a self-selected sample of journals from one publisher, we selected 11 journals from a range of biomedical specialties including two large general medical journals attracting submissions from around the world, with a combination of hybrid and open access journals with varying journal impact factors. Earlier studies looking at the effects of the pandemic on gender inequalities focused on the opening months and captured only the first international lockdowns; however, we have included submissions across the whole of 2020 until 31 May 2021, as multiple lockdowns occurred and international differences existed in policies and stipulations that were difficult to account for. We included a longer pandemic period and further divided the pandemic into four time periods to try to account for seasonal differences. We also tried to adjust for disparities between countries regarding lockdown policies and their impact on scientific production by including country as a random factor in our statistical analyses. We found the deepest fall in women's attainment of prominent authorship positions at the beginning of the pandemic (between 1 January and 31 May 2020), when most countries applied strict lockdown measures, including school and university closures. The urgent need for evidence on covid-19 during the pandemic led to a need for an expedited research and publication process. By identifying submissions related to covid-19, we were able to assess the real current impact of the pandemic and lockdown measures on gender disparities in research production and authorship on a prominent and competitive topic. However, our search strategy, similarly to others, ${ }^{21}$ to identify covid-19 relevant manuscripts was over- inclusive, as authors tended to refer to the pandemic to make their manuscripts more topical.

To minimise missing data and misclassification bias, we determined authors' gender by using a four step sequential procedure and set a high threshold for data accuracy within the software used. Although we were unable to determine gender for $19.3 \%$ of all authors across all submissions, we thought that using a high threshold was more important than getting more genders determined with a lower threshold for accuracy. We adjusted the models on gender missingness and did sensitivity analyses using accuracy of gender determination above 95\% and multiple imputation to replace missing gender determination data; both sensitivity analyses have confirmed our original results. However, by using software that determines gender in a binary form-namely, "male" and "female" based solely on name and country-we were unable to take into account the gender identity of individuals and may have misrepresented people with diverse gender identities. Further research in this area should try to integrate a broader definition of gender; however, for this to be conducted in large samples, these data will need to be self-reported routinely on journal submission systems. ${ }^{28}$ More generally, a greater need exists to evaluate the role of gender, ethnicity, and cultural diversity on many aspects of the production of science. ${ }^{29}$ Finally, we excluded duplicate manuscript submissions by identifying those that were transferred to another journal and removed those with an identical title and a submission date later than the original. We acknowledge that our method to identify duplicates may be imperfect, but it probably covers the vast majority of manuscripts, as automatic transfers use exactly the same manuscript title.

\section{Policy implications}

Academic promotion and awarding of research grants are heavily influenced by the publication records of candidates, and competition for prominent authorship positions (first, last, corresponding) is high. ${ }^{30}$ Studies of published research have already shown gender disparities, ${ }^{131-34}$ but our demonstration of even wider disparities at the submission stage during the pandemic suggests that we may observe even wider gender disparities in published research over the next few years once these manuscripts have reached publication stage. This may have important implications for career development, awarding of grant applications, and job opportunities for women.

During the covid-19 pandemic, women, particularly younger women, seem to have paid a heavier price than men from the lockdown measures and the need to reorganise work and research environments while assuming childcare, home schooling, housekeeping, and domestic work. ${ }^{5} 7$ 35-37 Female researchers have been less involved in research related to covid-19, and subsequently they have produced proportionately fewer research manuscripts, reflected by lower submission rates among female first authors compared with male first authors. ${ }^{7}$ In a survey conducted among 3345 Brazilian academics and research centres between 
April and May 2020, women with children, especially younger children, submitted significantly fewer research manuscripts than did men with children, with an even bigger difference compared with men without any children. This survey additionally showed that the intersection between race, gender, and parenthood has influenced academic productivity, with black female researchers being the most effected by covid-19. ${ }^{38}$ Furthermore, in general, teaching responsibilities are shouldered more by women faculty members than men, ${ }^{39}$ and women may have had even less time to write and submit research manuscripts during the pandemic as a result of the increased demands from the rapid shift to online teaching and an increase in mentorship duties and the need to support the mental health of students. ${ }^{3640}$ This slowdown in the race for scientific production will undoubtedly have practical implications for the near future. The consequences will be worse for young women researchers if adjustment measures are not rapidly implemented at academic institutions and competitive funding agencies.

People have proposed the introduction of voluntary disclosure of gender as part of the submission process to overcome the systematically low representation of women in authorship of scientific manuscripts and the application of gender quotas for the appointment to prominent academic positions to correct for gender disparities in the research environment. ${ }^{8} 41$ The systematic participation of all women researchers in mentorship programmes might also be an important area to develop because professional social networks seem to be more prominent among men than women. ${ }^{42} 43$ This might explain why male dominant environments lead to an amplification of homophily and why, despite the support of young female researchers by senior female researchers, gender inequalities in scientific production still persist. ${ }^{44} \mathrm{We}$ found that when a woman was last author, women were more likely to hold a prominent authorship position. The benefits of more senior women (reflected by last authorship position) supporting other women as first authors or more generally the promotion of female participation in research by other women has already been discussed and demonstrated by others. ${ }^{45-48}$ This mechanism of sisterhood promotion, which we observed in our study, has also been shown by others through a high correlation between the percentage of tenured female professors and the percentage of female full professors at universities. ${ }^{49}$ Implicit bias training may also help assessors in academia to evaluate women more equally for academic promotion. ${ }^{47}$ Finally, journals can also try to reduce gender bias by implementing policies to encourage gender balance in the editorial staff and advisory boards of journals, as well as among those invited to peer review manuscripts and author commissioned articles. ${ }^{5051}$

\section{Conclusions}

Patterns in authorship need to be routinely scrutinised to draw attention to the continued imbalance in female representation until parity can be reached.
The low rates of female first, last, and corresponding authorship on submissions may fall further as the pandemic continues to cause disruption to working patterns and may be compounded once the new research that was conceived and designed during the pandemic flows through to publication stage. Thus, our findings should give early warning of inequity to those who rely on authorship of published articles as a metric of productivity to inform academic promotion and award research grants.

We thank the editors of the participating journals for giving us access to the data. We thank Christophe Combescure and Antoine Poncet for their valuable input on modelling the number of female authors in the manuscripts.

Contributors: AGA and SS were involved in the study concept and design and in data collection and management. MR was involved in the gender determination of authors. KBM was responsible for data management. AGA was responsible for obtaining research funding. AGA and KBM were involved in data analysis and interpretation and in generating figures and tables. AGA, KBM, MR, and SS were involved in drafting and revising the manuscript. All authors approved the final version. AGA is the guarantor. The corresponding author attests that all listed authors meet authorship criteria and that no others meeting the criteria have been omitted.

Funding: This study was supported by grant 192374 from the Swiss National Science Foundation (SNF). The funders had no role in considering the study design or in the collection, analysis, or interpretation of data, the writing of the report, or the decision to submit the article for publication.

Competing interests: All authors have completed the ICMJE uniform disclosure form at www.icmje.org/disclosure-of-interest/ and declare: support from the university hospitals of Geneva, Geneva Medical School, and the SNF for the submitted work; SS and MR are full time employees of BMJ Publishing Group but are not involved in decision making on research submissions; no other relationships or activities that could appear to have influenced the submitted work.

Ethical approval: Ethical approval for the ATHENA project was received from the Institutional Review Board of the Geneva Canton on 16 April 2018 (No 2019-00540).

Data sharing: Relevant anonymised manuscript level data are available on reasonable request from the corresponding author. The study was conducted under a confidentiality agreement between the BMJ and the medical school of Geneva University, represented by the Department of Health and Community Medicine.

The guarantor affirms that this manuscript is an honest, accurate, and transparent account of the study being reported; that no important aspects of the study have been omitted; and that any discrepancies from the study as originally planned (and, if relevant, registered) have been explained.

Dissemination to participants and related patient and public communities: The results from this ancillary study to ATHENA broader research project will be presented both nationally and internationally at conferences and published in established peer reviewed journals. Dissemination of results to public communities will take place via media and on social network platforms from the affiliated institutions. Results will also be shared with the respective BMJ journal editors.

Provenance and peer review: Not commissioned; externally peer reviewed.

This is an Open Access article distributed in accordance with the Creative Commons Attribution Non Commercial (CC BY-NC 4.0) license, which permits others to distribute, remix, adapt, build upon this work non-commercially, and license their derivative works on different terms, provided the original work is properly cited and the use is noncommercial. See: http://creativecommons.org/licenses/by-nc/4.0/.

Filardo G, da Graca B, Sass DM, Pollock BD, Smith EB, Martinez MAM. Trends and comparison of female first authorship in high impact medical journals: observational study (1994-2014). BMJ 2016;352:i847. doi:10.1136/bmj.i847

2 Jagsi R, Guancial EA, Worobey CC, et al. The "gender gap" in authorship of academic medical literature--a 35-year perspective. $N$ Engl J Med 2006;355:281-7. doi:10.1056/NEJMsa053910

3 Bendels MHK, Müller R, Brueggmann D, Groneberg DA. Gender disparities in high-quality research revealed by Nature Index journals. PLoS One 2018;13:e0189136. doi:10.1371/journal.pone.0189136 
4 Gayet-Ageron A, Poncet A, Perneger T. Comparison of the contributions of female and male authors to medical research in 2000 and 2015: a cross-sectional study. BM Open 2019;9:e024436. doi:10.1136/bmjopen-2018-024436

5 Blasko Z, Papadimitriou E, Manca A. How will the COVID-19 crisis affect existing gender divides in Europe? 2020. https://ec.europa. eu/jrc/en/publication/eur-scientific-and-technical-research-reports/ how-will-covid-19-crisis-affect-existing-gender-divides-europe.

6 Brubaker L. Women Physicians and the COVID-19 Pandemic. IAMA 2020;324:835-6. doi:10.1001/jama.2020.14797

7 Myers KR, Tham WY, Yin Y, et al. Unequal effects of the COVID-19 pandemic on scientists. Nat Hum Behav 2020;4:880-3. doi:10.1038/s41562-020-0921-y

8 Pinho-Gomes A-C, Peters S, Thompson K, et al. Where are the women? Gender inequalities in COVID-19 research authorship. BM Glob Health 2020:5:e002922 doi:10.1136/bmigh-2020-002922

9 Yildirim TM, Eslen-Ziya $\mathrm{H}$. The differential impact of COVID-19 on the work conditions of women and men academics during the lockdown. Gend Work Organ 2020:28:243-9. doi:10.1111/gwao.12529

10 Williams WA2nd, Li A, Goodman DM, Ross LF. Impact of the Coronavirus Disease 2019 Pandemic on Authorship Gender in The Journal of Pediatrics: Disproportionate Productivity by International Male Researchers. J Pediatr 2021;231:50-4. doi:10.1016/j. jpeds.2020.12.032

11 DeFilippis EM, Sinnenberg L, Mahmud N, et al. Gender Differences in Publication Authorship During COVID-19: A Bibliometric Analysis of High-Impact Cardiology Journals. J Am Heart Assoc 2021;10:e019005. doi:10.1161/JAHA.120.019005

12 Andersen IP, Nielsen MW, Simone NL, Lewiss RE, Jagsi R. COVID-19 medical papers have fewer women first authors than expected. Elife 2020;9:e58807. doi:10.7554/eLife.58807

13 Muric G, Lerman K, Ferrara E. Gender Disparity in the Authorship of Biomedical Research Publications During the COVID-19 Pandemic: Retrospective Observational Study. J Med Internet Res 2021;23:e25379. doi:10.2196/25379

14 Lerchenmüller C, Schmallenbach L, Jena AB, Lerchenmueller MJ. Longitudinal analyses of gender differences in first authorship publications related to COVID-19. BMJ Open 2021;11:e045176. doi:10.1136/bmjopen-2020-045176

15 Brown C, Novick TK, Jacobs EA. Gender Disparities in Authorship of Invited Manuscripts During the COVID-19 Pandemic. Womens Health Rep (New Rochelle) 2021;2:149-53. doi:10.1089/whr.2021.0023

16 Cook J, Gupta M, Nakayama J, El-Nashar S, Kesterson J, Wagner S. Gender differences in authorship of obstetrics and gynecology publications during the coronavirus disease 2019 pandemic. Am J Obstet Gynecol MFM 2021:3:100268. doi:10.1016/j.ajogmf.2020.100268

17 Prunty M, Rhodes S, Mishra K, et al. Female Authorship Trends in Urology During the COVID-19 Pandemic. Eur Urol 2021;79:322-4. doi:10.1016/j.eururo.2020.11.017

18 Wehner MR, Li Y, Nead KT. Comparison of the Proportions of Female and Male Corresponding Authors in Preprint Research Repositories Before and During the COVID-19 Pandemic. JAMA Netw Open 2020:3:e2020335. doi:10.1001/jamanetworkopen.2020.20335

19 Quak E, Girault G, Thenint MA, Weyts K, Lequesne J, Lasnon C. Author Gender Inequality in Medical Imaging Journals and the COVID-19 Pandemic. Radiology 2021;300:E301-7. doi:10.1148/ radiol.2021204417

20 Bell ML, Fong KC. Gender Differences in First and Corresponding Authorship in Public Health Research Submissions During the COVID-19 Pandemic. Am I Public Health 2021:111:159-63. doi:10.2105/AJPH.2020.305975

21 Squazzoni F, Bravo G, Grimaldo F, Garcia-Costa D, Farjam M, Mehmani B. No Tickets for Women in the COVID-19 Race? A Study on Manuscript Submissions and Reviews in 2347 Elsevier Journals during the Pandemic. 2020 . https://ssrn.com/abstract=3712813. doi:10.2139/ssrn.3712813

22 Santamaría L, Mihaljević H. Comparison and benchmark of nameto-gender inference services. PeerJ Comput Sci 2018;4:e156. doi:10.7717/peerj-cs.156

23 White IR, Royston P, Wood AM. Multiple imputation using chained equations: Issues and guidance for practice. Stat Med 2011;30:37799. doi:10.1002/sim.4067

24 Holman L, Stuart-Fox D, Hauser CE. The gender gap in science: How long until women are equally represented?PLoS Biol 2018;16:e2004956. doi:10.1371/journal.pbio.2004956

25 Huang M-Z, Sun Y-C, Gau M-L, Puthussery S, Kao C-H. First-time mothers' experiences of pregnancy and birth following assisted reproductive technology treatment in Taiwan. J Health Popul Nutr 2019;38:10. doi:10.1186/s41043-019-0167-3

26 Larivière V, Ni C, Gingras Y, Cronin B, Sugimoto CR. Bibliometrics: global gender disparities in science. Nature 2013;504:211-3. doi:10.1038/504211a
27 Al-Gazali L, Valian V, Barres B, et al. Laboratory life: Scientists of the world speak up for equality. Nature 2013;495:35-8. doi:10.1038/495035

28 Nielsen MW, Stefanick ML, Peragine D, et al. Gender-related variables for health research. Biol Sex Differ 2021;12:23. doi:10.1186 s13293-021-00366-3

29 Nielsen MW, Alegria S, Börjeson L, et al. Opinion: Gender diversity leads to better science. Proc Natl Acad Sci U S A 2017;114:1740-2. doi:10.1073/pnas.1700616114

30 Guraya SY, Norman RI, Khoshhal KI, Guraya SS, Forgione A. Publish or Perish mantra in the medical field: A systematic review of the reasons, consequences and remedies. Pak J Med Sci 2016;32:15627. doi:10.12669/pims.326.10490

31 Waisbren SE, Bowles H, Hasan T, et al. Gender differences in research grant applications and funding outcomes for medical school faculty. J Womens Health (Larchmt) 2008;17:207-14. doi:10.1089/ jwh.2007.0412

32 Macaluso B, Larivière V, Sugimoto T, Sugimoto CR. Is Science Built on the Shoulders of Women? A Study of Gender Differences in Contributorship. Acad Med 2016;91:1136-42. doi:10.1097/ ACM.0000000000001261

33 van den Besselaar P, Sandström U. Gender differences in research performance and its impact on careers: a longitudinal case study. Scientometrics 2016;106:143-62. doi:10.1007/s11192-0151775-3

34 West JD, Jacquet J, King MM, Correll SJ, Bergstrom CT. The role of gender in scholarly authorship. PLoS One 2013;8:e66212. doi:10.1371/journal.pone.0066212

35 Ribarovska AK, Hutchinson MR, Pittman Qل, Pariante C, Spencer S. Gender inequality in publishing during the COVID-19 pandemic. Brain Behav Immun 2021;91:1-3. doi:10.1016/j.bbi.2020.11.022

36 Viglione G. Are women publishing less during the pandemic? Here's what the data say. Nature 2020;581:365-6. doi:10.1038/d41586020-01294-9

37 Gewin V. Pandemic burnout is rampant in academia. Nature 2021:591:489-91 doi:10.1038/d41586-021-00663-2

38 Staniscuaski F, Kmetzsch L, Soletti RC, et al. Gender, Race and Parenthood Impact Academic Productivity During the COVID-19 Pandemic: From Survey to Action. Front Psychol 2021;12:663252. doi:10.3389/fpsyg.2021.663252

39 Raj A, Kumra T, Darmstadt GL, Freund KM. Achieving Gender and Social Equality: More Than Gender Parity Is Needed. Acad Med 2019;94:1658-64. doi:10.1097/ACM.0000000000002877

40 Malisch JL, Harris BN, Sherrer SM, et al. Opinion: In the wake of COVID-19, academia needs new solutions to ensure gender equity. Proc Natl Acad Sci U S A 2020;117:15378-81. doi:10.1073/ pnas.2010636117

41 Vernos I. Research management: Quotas are questionable. Nature 2013:495:39 doi:10.1038/495039a

42 Ibarra H. Homophily and Differential Returns: Sex Differences in Network Structure and Access in an Advertising Firm. Adm Sci Q 1992;37:422-47. doi:10.2307/2393451.

43 Carr PL, Raj A, Kaplan SE, Terrin N, Breeze JL, Freund KM. Gender Differences in Academic Medicine: Retention, Rank, and Leadership Comparisons From the National Faculty Survey. Acad Med 2018;93:1694-9. doi:10.1097/ACM.0000000000002146

44 Feld SL. The Structured Use of Personal Associates* Soc Forces 1984;62:640-52. doi:10.2307/2578704

45 Richter KP, Clark L, Wick JA, et al. Women Physicians and Promotion in Academic Medicine. N Engl / Med 2020:383:2148-57. doi:10.1056/ NEJMsa1916935

46 Teede HJ. Advancing women in medical leadership. Med J Aust 2019:211:392-394 e1 doi:10.5694/mia2.50287

47 Pritlove C, Juando-Prats C, Ala-Leppilampi K, Parsons JA. The good, the bad, and the ugly of implicit bias. Lancet 2019;393:502-4. doi:10.1016/S0140-6736(18)32267-0

48 Madsen S, Andrade M. Unconscious Gender Bias: Implications for Women's Leadership Development. J Leadersh Stud 2018;12:62-7. doi:10.1002/jls.21566

49 Bilen-Green C, Froelich KA, Jacobson SW. The Prevalence of Women in Academic Leadership Positions, and Potential Impact on Prevalence of Women in the Professorial Ranks. 2008. https://journals.psu.edu/ wepan/article/view/58533.

50 Chatfield C, Cook S, Ladher N, et al. Ten steps to gender equity: The BMJ's resolutions. 2021. https://blogs.bmj.com/bmj/2021/03/08/ ten-steps-to-gender-equity-the-bmjs-resolutions/.

51 Clark J, Horton R. What is The Lancet doing about gender and diversity? Lancet 2019;393:508-10. doi:10.1016/S01406736(19)30289-2

Web appendix: Supplementary materials 